\title{
ANÁLISIS DE UN PROGRAMA MÉDICO-ASISTENCIAL PARA DIABETES (DIABETIMSS) MEDIANTE EL MÉTODO FODA
}

Rodríguez Torres, Alejandra ${ }^{1,2}$; Casas Patiño, Donovan ${ }^{1,2}$; Contreras Landgrave, Georgina ${ }^{3}$; Camacho Ruiz, Jaime Esteban ${ }^{3}$ y Andrade Galicia, Angelina ${ }^{4}$

${ }^{1}$ Médico Familiar. Instituto Mexicano del Seguro Social UMF 195. ${ }^{2}$ Centro Universitario Amecameca. Universidad Autónoma del Estado de México. ${ }^{3}$ Unidad Académica Profesional Amecameca, Universidad Autónoma del Estado de México. ${ }^{4}$ Jefe de Enseñanza. Instituto Mexicano del Seguro Social UMF 70.

Resumen: La diabetes mellitus (DM), es una enfermedad crónico degenerativa que implica un gran reto para los sistemas médico asistenciales. En México representa el 34\% del presupuesto de servicios sociales del país, y hablando de costos directos e indirectos estos van de 330 y 100 millones de dólares anuales respectivamente. Desde el 2008 se implementó un programa institucional para la prevención y atención dirigido al paciente diabético, nombrado Módulo DiabetIMSS, con un enfoque estructurado, integral y multidisciplinario. Este estudio consistió en realizar un análisis desde el enfoque sistémico, el cual implicó considerar los contextos dinámicos que moldean a los sistemas, así como las interacciones entre éstos, mediante el método FODA. Por último se realizó una matriz de estrategias a fin de contemplarlas como tácticas de mejora de este programa no sólo en beneficio del enfermo, sino de la familia y el colectivo.

Palabras clave: administración de los servicios de salud, estrategias, organización y administración.

Recibido: 11 Noviembre 2014. Aceptado: 22 Febrero 2014. Publicado: 15 Abril 2015.

Revista electrónica publicada por el Departamento de Farmacología de la Escuela de Medicina de la Universidad de Costa Rica, 2060 San José, Costa Rica. ${ }^{\circledR}$ All rights reserved. Licensed under a Creative Commons Unported License. 


\title{
ANALYSIS OF A MEDICAL CARE PROGRAM FOR DIABETES (DIABETIMSS) FODA METHOD
}

\begin{abstract}
Diabetes mellitus (DM) is a chronic degenerative disease that involves a major challenge for health care systems. In Mexico represents $34 \%$ of the budget for social services in the country, and speaking of these direct and indirect costs range from 330 to 100 million dollars per year respectively. Since 2008 an institutional program for the prevention and treatment targeted to the diabetic patient named DiabetIMSS Module, this involved a multidisciplinary patient-led approach with a diagnosis of diabetes. This paper conducted an analysis from the systemic approach which involved considering the dynamic contexts that shape systems as well as the interactions between them, using the SWOT method. Finally a strategy matrix was performed in order to consider them as strategies for improvement of this program will not only benefit the patient, but the family and the collective.
\end{abstract}

Key words: health services administration, organization and administration, strategies.

\section{DIABETES MELLITUS: LA PROBLEMÁTICA}

La diabetes mellitus (DM), es una enfermedad crónico degenerativa que implica un gran reto para los sistemas médico asistenciales. La Organización Mundial de la Salud indica que de 1995 a la fecha casi se ha triplicado el número de personas diabéticas y cada día el riesgo de presentar diabetes aumenta [1,2]. En México, la edad promedio de las personas que murieron por diabetes en 2010 fue de 66.7 años, lo que sugiere una reducción de 10 años a la esperanza de vida general [3]. Las pruebas demográficas y epidemiológicas indican que si no se da una intervención eficaz, la frecuencia de la diabetes seguirá aumentado no solo en nuestro país sino en todo el orbe [4]. Este problema es aún mayor ya que deriva no sólo en el deterioro de la calidad de vida de las personas que la padecen con la consecuente pérdida de años de vida productiva y muerte, sino también en la demanda y el consumo de servicios hospitalarios, por lo que los costos de tratamiento son altos para la economía de las instituciones del Sistema nacional de salud. Se ha demostrado que el costo de la atención médica de las personas con diabetes puede ser entre 2-3 veces mayor que el de la población no afectada por la diabetes, esto significa que $25 \%$ de los gastos médicos se destinan para tratar la enfermedad, otro $25 \%$ para complicaciones y $50 \%$ se consume para la asistencia médica general. En México representa el 34\% del presupuesto de servicios sociales del país, y hablando de costos directos e indirectos estos van de 330 y 100 millones de dólares anuales respectivamente. Tan sólo para el Instituto Mexicano del Seguro Social (IMSS) su atención ocupa entre el 14-15\% de los recursos anuales destinados a salud $[5,6,7]$.

Los estilos de vida poco saludables son altamente prevalentes entre niños, adolescentes y adultos mexicanos, propiciando un aumento importante de la obesidad y sobrepeso, principal factor modificable de la diabetes, lo cual nos conlleva a 
retos aún mayores para alrededor de 5664870 niños en edad escolar (de 5 a 11 años de edad) con sobrepeso y obesidad a nivel nacional quienes en años no muy lejanos serán grupo blanco para este padecimiento [8]. Cabe mencionar que en 1993 la prevalencia de los diabéticos con diagnóstico conocido en población mayor de 20 años fue de 4.0 $\%$ mientras que en 2000 y 2007 se describió una prevalencia del 5.8 y $7 \%$, respectivamente [9,10], lo cual nos indica que la población altamente vulnerable será aquella en edad económicamente activa y reproductiva conllevando un mayor gasto no sólo en su diagnóstico y tratamiento oportuno, sino en sus complicaciones laborales, familiares, económicas y sociales implícitas que serían insostenibles para los sistemas de salud nacionales e internacionales.

\section{DIABETES MELLITUS: CIFRAS EN EL INSTITUTO MEXICANO DE SEGURO SOCIAL}

El sistema público de salud está constituido por instituciones de seguridad social: Instituto Mexicano del Seguro Social (IMSS), Instituto de Seguridad y Servicios Sociales de los Trabajadores del Estado (ISSSTE), Petróleos Mexicanos (Pemex), Secretaria de la Defensa (SEDENA), Secretaria de Marina (SEMAR).

Cabe mencionar que el mayor porcentaje de población diabética está siendo atendida en el IMSS. De cada cien personas que son atendidas en México, 47 son atendidas en el IMSS y 36 por la Secretaria de Salud (SSA) [11,12], lo cual representa aproximadamente 47 millones de mexicanos derechohabientes [13]. La DM constituye un reto creciente y transcendente para el IMSS, porque:

- Durante el periodo 2004-2012 la DM fue la primera causa de muerte con 21,096 defunciones en el año 2011 [16,17].

- Ocupa el octavo lugar de urgencias y como motivo de egreso hospitalario [18].
- 5 de cada 10 pacientes en los programas de diálisis son diabéticos tipo 2 [19].

- Es primera causa de los dictámenes de invalidez que corresponde el $14.3 \%$ del total, con una relación hombre mujer 8 a 1 (83 y 17\% respectivamente) [20].

- La DM es un factor de riesgo cardiovascular, se estima que entre 7 y 8 de cada 10 personas con diabetes mueren por este motivo [21].

- Es la principal causa de ceguera, amputación no traumática de miembros inferiores [22].

- Es la principal causa de pensión por invalidez (14.7\% del total) con una relación de hombre mujer de 6 a 1 [20)].

\section{MÓDULO DIABETIMSS: PROGRAMA PARA LA PREVENCIÓN Y ATENCIÓN DEL PACIENTE DIABÉTICO}

Desde el 2008 el IMSS, implementó en las Unidades de Medicina Familiar, un programa institucional para la prevención y atención dirigido al paciente diabético, nombrado Módulo DiabetIMSS, en los que se proporciona atención (médico-asistencial) con un enfoque estructurado, integral $y$ multidisciplinario dirigido al paciente con diagnóstico de diabetes; esto para la prevención de complicaciones, limitación del daño y rehabilitación, con el objetivo de lograr conductas positivas y cambios de estilo de vida saludables, buscando la corresponsabilidad del paciente y su familia [23,24]; los criterios de ingreso son: ser portador de cualquier tipo de diabetes: que acepte ser referido al módulo; con menos de 10 años de evolución, sin complicaciones crónicas y con más de 10 años de evolución, sin complicaciones crónicas; sin deterioro cognitivo, psicosis o fármaco dependencia; con compromiso de red de apoyo familiar o social; que pueda acudir una vez al mes para su atención integral que incluye una 
sesión educativa con una duración de 2 a 2.5 hrs. y una consulta médica mensual.

Actualmente se cuenta con 135 módulos [12], donde se han otorgado $1,130,682$ consultas a 141,475 pacientes, obteniendo un promedio de 2.73 pacientes atendidos por hora.

\section{OBJETIVO}

Presentar el análisis FODA del programa institucional DiabetIMSS y describir los contextos en que se brinda la consulta médico-asistencial a los derechohabientes, así como las principales características y problemas que se presentan en los procesos médico-asistenciales condicionados por el sistema originalmente planteado.

\section{MÉTODO}

Se realizó un análisis desde el enfoque sistémico el cual implicó considerar los contextos dinámicos que moldean a los sistemas, así como las interacciones entre éstos. El diagnóstico situacional FODA es una herramienta que posibilita conocer y evaluar las condiciones de operación reales de una organización, a partir del análisis de cuatro variables principales, con el fin de proponer acciones y estrategias para su beneficio [25].

\section{ANÁLISIS DEL PROGRAMA}

Teniendo como marco la problemática internacional y nacional de la diabetes mellitus y conociendo la dinámica de un programa institucional de educación y autocuidado como es el DiabetIMSS, generamos la siguiente pregunta: ¿Cumple el DiabetIMSS con sus objetivos planteados?

El método FODA (siglas de fortalezas, oportunidades, debilidades y amenazas), consiste en realizar una evaluación de los factores fuertes y débiles que, en su conjunto, diagnostican la situación interna de una organización, así como su evaluación externa, es decir las oportunidades y amenazas [25].
El análisis contempla dos ambientes, uno interno el cual se refiere a aquellos elementos que se relacionan directamente con la estructura y operación de la organización que incluye los recursos disponibles, áreas funcionales, actividades, etc.) en el cual se ubican las fortalezas y debilidades de la organización. Por otro lado el ambiente externo está integrado por componente sumamente amplios que se asocian también con variables de influencia global, como la economía, política, cultura, tecnología, políticas públicas, etc., por mencionar algunas; dentro de la cual se pueden observar tanto las oportunidades como las amenazas [25,26,27].

Antes de abordar el análisis conviene establecer los conceptos de las variables:

Fortaleza: Es algo en lo que la organización es competente, con elementos o factores que estando bajo su control mantiene un alto desempeño, generando ventajas o beneficios presentes y claros, con posibilidades atractivas a futuro.

Debilidad: Significa una deficiencia o carencia, algo en lo que la organización tiene bajos niveles de desempeño y por tanto es vulnerable. Constituye un obstáculo para la consecución de los objetivos, aun cuando esté bajo el control de la organización.

Oportunidades: Son aquellas circunstancias del entorno que son potencialmente favorables para la organización y pueden ser cambios o tendencias que se detectan y que pueden ser utilizados ventajosamente para alcanzar o superar los objetivos.

Amenazas: Son factores del entorno que resultan en circunstancias adversas que ponen en riesgo alcanzar los objetivos establecidos, pueden ser cambios o tendencias que se presentan repentinamente o de manera paulatina, las cuales crean una condición de incertidumbre e inestabilidad en donde la organización tiene muy poca o nula influencia. 
Para analizar el programa DiabetIMSS partamos de su medio externo y su medio interno.

\section{DEL MEDIO AMBIENTE EXTERNO: OPORTUNIDADES Y AMENAZAS}

En general tanto las amenazas como las oportunidades podrían quedar agrupadas en las siguientes categorías: factores económicos, sociales, políticos, tecnológicos, demográficos, etc.

\section{Oportunidades del DiabetIMSS:}

01. Mejorar las acciones de prevención y atención integral de la diabetes en el IMSS.

02. Proporcionar servicios de atención médica con el fin de promover, proteger y restaurar la salud, que permitan mejorar el control metabólico y prevenir o retrasar la aparición de complicaciones y de rehabilitación.

03. Fomentar la corresponsabilidad del paciente y su familia para la prevención, tratamiento, control y rehabilitación.

04. Desarrollar en forma permanente el sistema de información, gestión clínica y de recursos. En el mes de agosto de 2009 se publicó en Intranet la Guía de Práctica Clínica, “Diagnóstico y Tratamiento de la Diabetes Mellitus Tipo 2", misma que fue difundida a todas las Unidades de Medicina Familiar (UMF), en conjunto con otros documentos normativos que complementan la buena práctica para la atención del paciente diabético.

05. Otorgar atención médica a la población derechohabiente del IMSS diagnosticados con diabetes, basada en evidencia científica y que se traduzca en:

06. Mejorar el control metabólico. Programación de presupuesto a fin de que todas las unidades contaran, para el seguimiento de las metas de control metabólico y la identificación de nefropatía temprana, con los estudios de laboratorio para la cuantificación de hemoglobina glucosilada, colesterol , triglicéridos y microalbuminuria .

07. Prevenir o retrasar las complicaciones. Se incluyó en el Cuadro Básico Sectorial e Institucional la cámara no midriática de retina, recurso tecnológico necesario para identificar oportunamente las lesiones oculares en los pacientes diabéticos; mientras que el software de auto interpretación se encuentra en proceso de inclusión [13]. Introducción de equipo médico para la identificación de neuropatía periférica.

08.Mejorar el nivel de salud.

09. Propiciar satisfacción en los pacientes. Se remodeló o construyó infraestructura para la conformación de módulos. Se incorporaron 580 plazas de nueva creación de médicos familiares, enfermeras generales, nutricionistas $y$ trabajadoras sociales [23].

010. Propiciar el uso eficiente de los recursos. Por otra parte, con el fin de automatizar las actividades de salud desarrolladas en el programa se activó la aplicación DIABETIMSS en el Expediente Clínico Electrónico (ECE) de todas las Unidades de Medicina Familiar [13].

\section{Amenazas del DiabetIMSS:}

A1. El actual modelo y arquitectura de las unidades de atención primaria del IMSS no se ha modificado sustancialmente desde hace más de 30 años, y los criterios para determinar el número de médicos, enfermeras, trabajadoras sociales o nutriólogos no se han revisado; el modelo fue diseñado para la atención de una población con predominio de enfermedades agudas y no responde al cambio epidemiológico.

A2. El gasto total de un día para la atención de pacientes diabéticos que reciben atención en el IMSS es de 45,123,738 pesos, correspondiendo a la atención ambulatoria 31'374,410 pesos (69.5 por ciento); a la hospitalaria, $10,916,041$ pesos $(24.2$ por ciento); a las sesiones de hemodiálisis 
ambulatoria, 850,592 pesos (1.9 por ciento); a las sesiones de diálisis peritoneal, 1'254,740 pesos (2.8 por ciento), y a la rehabilitación de pacientes amputados, 727,955 pesos (1.6 por ciento). Los impactos económicos esperados entre los cinco y siete años se relacionan con la disminución de 15 por ciento en los costos, $20 \%$ en complicaciones, $18 \%$ en invalidez y $14 \%$ en mortalidad [13].

A3. El promedio del consumo diario de calorías "por los mexicanos" se ha incrementado, mientras que la actividad física ha disminuido: a principios del siglo XX, más de $70 \%$ de la población se dedicaba a las actividades primarias (como la agricultura), que demandan un importante esfuerzo físico; ahora, más de la mitad de la población se emplea en la prestación de servicios (actividades sedentarias) [28].

A4. En 12 años, el gasto promedio para adquirir frutas y vegetales se redujo casi $30 \%$ mientras que el dedicado a comprar bebidas embotelladas aumentó cerca de $40 \%$. Las consecuencias son evidentes: en la Encuesta Nacional de Salud y Nutrición Alimentación (ENSANUT) del 2000, 60 \% de los adultos fueron clasificados con sobrepeso $u$ obesidad; mientras que en la ENSANUT del 2005 esta proporción creció a 69 \% [28].

\section{DEL MEDIO AMBIENTE INTERNO:}

\section{FORTALEZAS Y DEBILIDADES}

Las demandas del medio ambiente externo sobre la institución, deben ser cubiertas con los recursos de la organización. Las fortalezas y debilidades internas varían considerablemente para diferentes instituciones, sin embargo, pueden muy bien ser categorizadas en: a) administración y organización, b) operaciones, 3) finanzas y 4) otros factores específicos para la institución.

\section{Fortalezas del DiabetIMSS:}

F1. Existen 136 módulos DiabetIMSS en todo el país donde son atendidos 103 mil pacientes, de los que el 48 por ciento está controlado.
F2. Además de consultas médicas y manejo farmacológico, quienes padecen la enfermedad reciben sesiones educativas para cambiar sus estilos de vida.

F3. La atención convencional consiste en una consulta mensual con el médico familiar quien se apoya en el equipo de salud: trabajo social, nutrición y medicina preventiva. Las evaluaciones del modelo convencional indican que los pacientes no reciben la atención que requieren; por ejemplo, únicamente $3.9 \%$ recibe orientación nutricional y a $30 \%$ le han revisado los pies $[13,29,30]$.

F4. El programa proporciona atención (médicoasistencial) con un enfoque estructurado, integral y multidisciplinario que está dirigido al paciente con diagnóstico de DM2, para la prevención de complicaciones, limitación del daño y rehabilitación, con el objetivo de lograr conductas positivas y cambios a estilos de vida saludables, buscando la corresponsabilidad del paciente y su familia [24].

\section{Debilidades del DiabetIMSS:}

D1. Tiene escasa coordinación de sus integrantes, sobrecarga laboral y duración insuficiente de la consulta médica, factores que influyen negativamente en la calidad de la atención, satisfacción de los pacientes y resultados en su salud; únicamente 30\% logra control metabólico.

D2. La estimación de los recursos se realizó mediante el método de planificación ajustado por objetivos de los servicios y basado en la evidencia sobre las mejores prácticas de atención. Para ello se determinaron: a) la capacidad de producción de consultas por trabajador por año, b) las consultas programadas para la atención a pacientes con diabetes por año y c) el número total de recursos humanos necesarios en función del número de consultas programadas al año. El cálculo se realizó para cada modelo de atención (convencional y DiabetIMSS) tomando en cuenta tres escenarios: a) atención de pacientes con diabetes de acuerdo con 
la frecuencia recomendada actualmente por el IMSS: 12 consultas al año con médico y con enfermera y seis consultas al año con nutriólogo y trabajadora social, independientemente del estado metabólico del paciente. b) atención de acuerdo con la frecuencia recomendada por los expertos (para pacientes con diabetes descontrolados: 12 consultas al año con médico y con enfermera y seis consultas al año con nutriólogo y trabajadora, y para pacientes controlados: seis consultas al año con médico y con enfermera y una consulta al año con nutriólogo y trabajadora social, considerando la prevalencia de pacientes descontrolados de $70 \%$; [13,29] y c) atención con los mismos parámetros del escenario $b$, pero considerando un escenario con una prevalencia de pacientes controlados de $50 \%$.

D3. El tiempo promedio requerido para otorgar consulta fue el siguiente: médico, 35 minutos; enfermera, 25 minutos; nutrióloga y trabajador social, 40 minutos, respectivamente por expertos [31]. El análisis consideró la estimación del tiempo (en horas) que en un año laboral el personal de salud dedica a la atención de pacientes con diabetes, asumiendo que, además requieren atender pacientes por otros motivos de consulta. En el modelo convencional, un médico familiar dispone de 339 horas para la consulta a pacientes con diabetes, una enfermera 87, una nutrióloga 506 y un trabajador social 69 horas.

D4. En el modelo convencional las consultas del médico familiar y la enfermera tienen una duración de 15 minutos, respectivamente, mientras que en DiabetIMSS el promedio es de 16 minutos. Las consultas que la nutrióloga y el trabajador social otorgan tienen una duración promedio de 15 minutos en ambos modelos. Los resultados indican que hay discrepancia importante entre la cantidad recomendada de tiempo por consulta (35 minutos) y la que actualmente se destina a los pacientes. El médico dedica 15 minutos y su déficit es de 20 minutos.

D5. El médico familiar tiene limitaciones para realizar actividades educativas en función de sus Revista electrónica publicada por el Departamento de Farmacología de la Escuela de Medicina de la Universidad de Costa Rica, 2060 San José, Costa Rica. ${ }^{\circledR}$ All rights reserved. Licensed under a Creative Commons Unported License. demandas de tiempo, puesto que se encuentra inmerso en ambientes en los que predomina el burocratismo, el autoritarismo, la regulación en exceso y la gestión centrada en el control, con la consecuente falta de motivación, rigidez y trabajo rutinario, que hacen que deje de lado su función primordial [32].

D6. Los pacientes son citados mensualmente para recibir atención. Este criterio es de carácter administrativo y obedece principalmente a la necesidad de resurtir la receta o de indicar estudios de laboratorio.

D7. La educación grupal debe de ser lo más completa posible, y contemplar las necesidades de los pacientes, y de acuerdo a su disponibilidad de tiempo. Al avanzar la edad hay una pérdida progresiva de los recursos físicos y mentales que tiende a producir sentimientos de vulnerabilidad, que de igual manera tendríamos que considerar al tratar a grupos ancianos [34].

D8. No existen estudios que evalúen el impacto de este programa en los 5 años siguientes a su formación, sino son estudios con evaluaciones a máximo un año, que tratándose de una enfermedad crónica no tiene un impacto definido $[18,34,35,36]$.

D9. De acuerdo con la opinión de algunos pacientes inscritos en el programa, el módulo no cuenta con el personal necesario para garantizar el correcto seguimiento y control de la enfermedad, el responsable de impartir las referidas pláticas ha sido sustituido en varias ocasiones y se hace uso de personal improvisado en el tema, y con temas repetitivos [37].

D10. El tiempo promedio de estancia en el módulo fue de 10.24 meses. El principal motivo de retiro fue decisión personal 43.3\%, problemas administrativos y laborales $40 \%$, limitaciones físicas $10 \%$ y cambio de residencia 6.6\% [38].

D11. Existe un sub registro de las actividades por los responsables de cada servicio, falta de insumos

61 
y personal en el módulo, comunicación ineficaz entre los profesionales que operan en el módulo, técnicas didácticas no adecuadas a la población, pérdida de derechohabiencia $y$ horarios establecidos [38].

\section{MATRIZ FODA DEL DIABETIMSS}

Una vez que tenemos determinada las categorías del método FODA en un primer plano, se realizó una asociación entre ellas teniendo como referencias la Misión y la Visión del programa DiabetIMSS. Al final la matriz FODA (Figura 1) nos indica cuatro estrategias alternativas conceptualmente distintas, tal como lo propone David (39).

\section{ESTRATEGIAS}

\section{1.- Estrategia Maxi- Maxi (Fortalezas y Oportunidades)}

a) Consolidar un grupo transdisciplinario médicoasistencial, educativo, preventivista, que incida más allá del modelo terapéutico y despierte conciencia individual, familiar y colectiva para el combate a la diabetes, fomentando la participación paciente-familia en el proceso de la saludenfermedad para la búsqueda de mejores estilos de vida y promoción de la salud y mejorar la calidad de vida. $(01,02,03,08, F 1, F 2, F 3, F 4, F 5)$.

b) Analizar y actualizar los criterios de evaluación y los indicadores de monitoreo de la función del programa DiabetIMSS, tomando en consideración la satisfacción de los pacientes y las necesidades de los recursos humanos para desempeñar su actividad. (04, 05, 08, 09, F2, F3, F4).

Figura 1. Matriz FODA

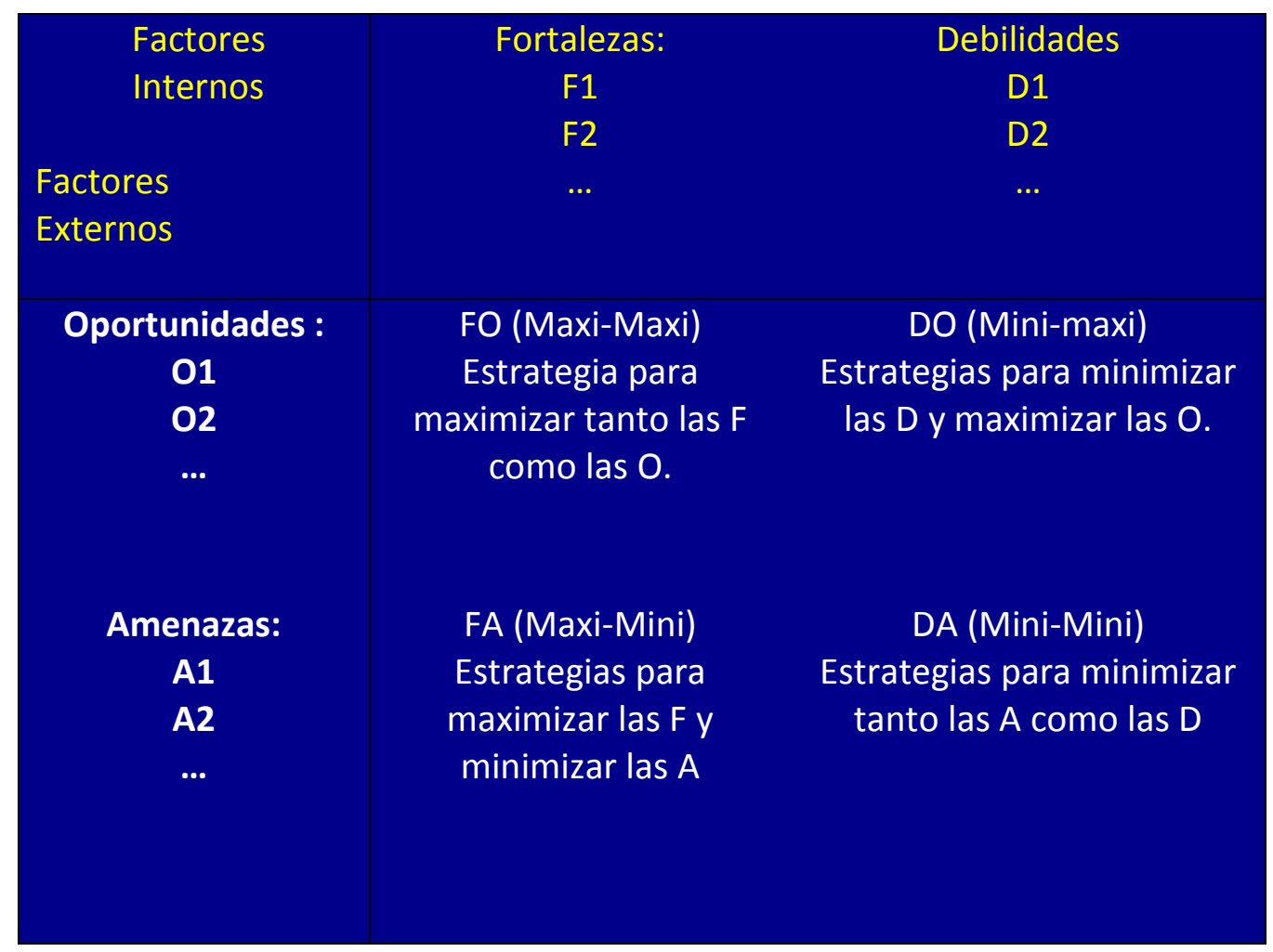

Fuente :Elaboración propia

Revista electrónica publicada por el Departamento de Farmacología de la Escuela de Medicina de la Universidad de Costa Rica, 2060 San José, Costa Rica. ${ }^{\circledR}$ All rights reserved. Licensed under a Creative Commons Unported License. 


\section{2.- Estrategia Maxi-Mini (Fortalezas y Amenazas):}

a) Enfatizar en el modelo preventivista del programa y no seguir perpetuando el modelo curativo, motivando mayor participación de la familia como grupo vulnerable y no sólo atender las necesidades medicamentosas del paciente, bajo la guía de un grupo multidisciplinario.

b) Tener un mayor acercamiento a los usos y costumbres a fin de abatir con la obesidad y los condicionantes sociales que la acompañan,

incorporando a la familia en el problema de la diabetes como generadora de usos y costumbres de cómo visualizar y vivir la diabetes. (A3, A4, A5, F1, F4, F5).

\section{3.- Estrategia Mini-Maxi (Debilidades y Oportunidades)}

a) Reestructurar la formación de los grupos considerando el tipo de población ya que de la caracterización de estos, se requerirán diferentes metodologías educativas, asistenciales, de apoyo, etc. $(01,02$, D2, D3, D4).

b) Generar sentido de pertenencia e identidad en los grupos a fin de que ellos fortalezcan redes de apoyo con sus semejantes y con su familia, e inicien con modificaciones en sus estilos de vida que se puedan transpolar a otros grupos. (01, 02, 03, D3, D8).

\section{4.- Estrategia DA (Mini-Mini)}

a) Garantizar que el programa cuente con los recursos humanos y materiales que planteó de acuerdo a los objetivos del programa en todos los módulos formados. Capacitación del personal tomando en consideración puntos clave como métodos de enseñanza, manejo de grupos focales, considerar el factor cultural, el nivel educativo, usos y costumbres, teniendo en consideración que los adultos mayores requieren de mayor tiempo de atención médico-asistencial y las técnicas educativas también requieren ser diferentes que pacientes más jóvenes por cuestiones de retención, memoria y o discapacidades auditivas y o visuales. (A1, A3, A4,A5, D2,D3,D4).

b) Se debe de tener una muy adecuada comunicación entre los profesionales de salud que integran el modulo, para garantizar que la información otorgada por cada uno de ellos siga una misma sintonía y no se contraponga un discurso contra otro. Enfatizando el papel informativo-preventivo del personal y no sólo cumplir con asistir a la cita como un compromiso administrativo para adquirir una receta. (A1, A3, A4, A5, D1, D2, D9,D10).

c) El personal deberá entregar un informe que no se preste a sub-registro de actividades o logros de los indicadores solicitados, ya que el hecho de brindar informes tendenciosos a las metas establecidas, contrapone los objetivos establecidos en el programa, como el control glicémico, el diagnóstico de complicaciones de pies, ojos etc. (A1,A2,D2,D11)

\section{DISCUSION}

La mirada nosológica proyectada al campo del cuidado de la salud ha permitido un impresionante desarrollo de la prevención, el diagnóstico, el tratamiento y la rehabilitación de las enfermedades en beneficio de millones de personas. No obstante, las limitaciones de tal aproximación se hacen ostensibles cuando nos acercamos más al ámbito de lo cultural: a los usos y costumbres, a los hábitos, tradiciones y creencias de las personas, a sus condiciones y circunstancias de vida, sobre todo en una enfermedad crónica degenerativa como la diabetes, la cual está fuertemente relacionada con la obesidad.

\section{CONCLUSIONES}

De los métodos y técnicas de diagnóstico aplicables en las organizaciones, la matriz FODA permite evaluar las fortalezas, oportunidades, debilidades y amenazas, destacando el procedimiento a seguir para su análisis y derivación de estrategias para su enriquecimiento. Es recomendable utilizar la 
metodología que garantice su aplicación en escenarios y campos de conocimiento académico a efecto de justificar las adecuaciones, mejoramientos, reformas, modernizaciones 0 transformaciones de los modelos para realizar diagnósticos y determinar estrategias de intervención en las organizaciones productivas y sociales.

La DM representa un gran reto a corto y mediano plazo para los sistemas de salud no tanto por la atención de complicaciones agudas y crónicas, sino para detener la presentación de la enfermedad en grupos familiares y en edades cada vez más jóvenes, por lo que grupos de autoayuda o focales como el DiabetIMSS son muy importantes y tendrán un impacto positivo en dicha tarea siempre y cuando se considere en primer instancia las necesidades reales de los usuarios y la disponibilidad del sistema de salud en ofertar los servicios. El primer nivel de atención es una pieza clave para este tipo de enfermedades crónico degenerativas, su mayor importancia radica en la promoción de cambios de estilo de vida y la prevención, su carácter informativo, educativo y de promoción requiere estar apegada a los determinantes sociales, culturales, económicos, educativos para promocionar la participación individual, familiar y colectiva en virtud de generar una cooperación dentro de la atención médica y no únicamente una acción pasiva como lo ha motivado la práctica médica hegemónica curativa. El programa institucional de DiabetIMSS ha mostrado tener impactos positivos en el manejo y control de la diabetes, sin embargo, requiere considerar algunas modificaciones dentro de su contenido y estructura a fin de optimizar los resultados no sólo en el enfermo, sino también en la familia y en el colectivo.

\section{BIBLIOGRAFÍA}

1. Organización Mundial de la Salud. Nota descriptiva No. $132 . \quad$ OMS. http://www.who.int/mediacentre/factsheets/fs312 /es/index.html. Accesada 17 diciembre de 2012).
2. International Diabetes Federation. Atlas de Diabetes. http://www.idf.org/diabetesatlas/5e/Update2012. Accesada 17 diciembre de 2012.

3. Instituto Nacional de Estadística y Geografía. Cuéntame..... Población. http://cuentame.inegi.org.mx/poblacion/esperanza. aspx?tema=P. Accesada 17 de marzo de 2014.

4. Asociación Latinoamericana en diabetes. Simposium sobre economía y diabetes. http://www.paho.org/spanish/ad/dpc/nc/dia-aladbackg. Accesada 19 de febrero 2013.

5. Arredondo A. Diabetes: a global challenge with high economic burden for public health systems and society. American Journal of Public Health.2013; 103(2):1-2.

6. PROFECO. Brújula de compra de Profeco. http://profeco.gob.mx/encuesta/brujula/bruj_2007 /bol47_diabetes.as. Accesada 02 de marzo 2013.

7. Instituto Nacional de Salud Pública. Encuesta Nacional de Salud 2012. http://ensanut.insp.mx/informes/ENSANUT2012Re sultadosNacionales.pdf. Accesada 13 de febrero 2013.

8. Rivera J. Sobrepeso y Obesidad en niños y adolescentes. En: Encuesta Nacional de Salud y Nutricion. Gutiérrez J (ed). Instituto Nacional de Salud Pública, Cuernavaca, México, 2012. pp 132133.

9. Aguilar CA. Prevalence and characteristics of early onset type 2 diabetes in Mexico. Am J Med. 2002; 13(7): 569-574.

10. Villalpando S. Trends for type 2 diabetes and other cardiovascular risk factors in Mexico from 19932006. Salud Pública Mex. 2010; S72-S79.

11. Instituto Nacional de Estadística, Geografía e Informática.

http://www.inegi.org.mx/.../estadísticas/2010/diab etes0.doc. Accesada 16 diciembre 2012.

12. Dirección de Prestaciones Médicas IMSS. Diagnóstico y tratamiento de la diabetes mellitus tipo 2 . http://www.imss.gob.mx/profesionales/guiasclinica s/Documents/000GER Diabetes Mellitus.pdf. Accesada el 18 de febrero 2013.

13. Instituto Mexicano del Seguro Social. Informe al Ejecutivo Federal y Congreso de la Unión sobre la

Revista electrónica publicada por el Departamento de Farmacología de la Escuela de Medicina de la Universidad de Costa Rica, 2060 San José, Costa Rica. ${ }^{\circledR}$ All rights reserved. Licensed under a Creative Commons Unported License. 
situación financiera y riesgos del Instituto Mexicano del Seguro Social 2011-2012. México: IMSS.

14. Membreño P, Zonana A. Hospitalización de pacientes con diabetes mellitus. Causas, complicaciones y mortalidad. Rev Med IMSS. 2005; 43(2): 97-101.

15. García G, Díaz R. Diabetes mellitus 2: autoconcepto, evolución y complicaciones. Desarrollo Cientif Enfer. 2005; 13(7): 207-210.

16. Sistema Nacional de Información en Salud. Programa de Acción Específico 2007-2012. Secretaría de Salud. 2008. México; 7-83.

17. Gutiérrez G, Flores S, Fernández I, Martínez O, Velazco V, \& et al. Estrategia de prestación y evaluación de servicios preventivos. Rev Med IMSS. 2006; 44: S3-S21.

18. León M, Araujo G, Linos Z. DiabetIMSS: eficacia del programa de educación en diabetes en los parámetros clínicos y bioquímicos. Rev Med Inst Mex Seg Soc. 2012; 51(1): 74-79.

19. Dirección de Prestaciones Médicas. Reporte de actividades prestadas por el IMSS, período 2011. http://www.imss.gob.mx/estadisticas/Documents/2 0112012/C12.pdf. Accesada 12 de enero 2013

20. Méndez D, Méndez B. Epidemiología de la insuficiencia renal en México. Dial Traspl. 2010; 30(1): 7-11.

21. Gil LS. Perspectiva de la diabetes mellitus tipo 2 en el Instituto Mexicano del Seguro Social. Rev Med Inst Mex Seguro Social. 2013; 51(1): 58-67.

22. IMSS. Guía técnica para Otorgar Atención médica en el módulo de DiabetIMSS a derechohabientes con diagnóstico de diabetes mellitus en Unidades de Medicina Familiar. 2009

23. Ponce T. La matriz FODA: alternativa de diagnóstico y determinación de estrategias de intervención en diversas organizaciones. Enseñanza e investigación en psicología; 2007 12(1): 113-130.

24. Ramírez J. Material del curso: Gestión estratégica, Maestría en Ciencias Administrativas. 2007. IIESCA UV, México.

25. Comité de Educación en Diabetes Fundación IMSS, A.C. El papel del derechohabiente en la prevención y control de la diabetes mellitus. Rev Med Inst Mex Seguro Social. 2007; 45(2): 101-103.
26. Pérez R. Evaluating quality of care for patients with type 2 diabetes using electronic health record information in Mexico. BMC Med Inform Decis Mak. 2012; 12(50).

27. Salinas AM. Technical efficiency in primary care for patients with diabetes. Salud Pública Méx. 2009; 51: 48-58.

28. Douviva S. Recursos humanos para la atención de pacientes con diabetes en unidades de medicina familiar del Instituto Mexicano del Seguro Social. Salud Pública Méx. 2013; 55(6): 607-617.

29. López A., B. L. Control clínico posterior a sesiones grupales en pacientes con diabetes e hipertensión. Rev Ins Mex Seguro Soc. 2007; 45(1): 29-36.

30. Mazón M. (2012). Eficacia del programa de educación en diabetes en los parámetros clínicos y bioquímicos. Rev Med Ins Mex Seguro Social. 2012; 51(1): 74-79.

31. Figueroa S, Cruz JE, Ortiz AR, Lagunes AL, Jiménez J, Rodríguez JR. Estilo de vida y control metabólico en diabéticos del programa DiabetIMSS. Gaceta Médica de México. 2014; 150:29-34.

32. Mendoza MV. Impacto de un programa institucional educativo en el control del paciente diabético. Rev Med Inst Mex Seguro Soc.2013; 51(3), 254-259.

33. Memorias del XXI Congreso Nacional de Medicina Familiar. http://www.facmed.unam.mx/deptos/familiar/15(2 ).pdf. Accesada el 18 de febrero 2013.

34. Martínez, G. Insuficiente el personal en módulos de DiabetIMSS.

http://www.oem.com.mx/elsoldecuautla/notas/n32 86086.htm. Accesada el 10 de febrero 2014.

35. Zúñiga Ma. Perfil de uso de los servicios del módulo DiabetIMSS por pacientes con diabetes mellitus tipo 2. Rev Enferm Inst Mex Seguro Soc. 2013; 21(2), 7984.

36. David, F. (1997). Conceptos de administración estratégica. México: Prentice-Hall Hispanoamericana.

\section{INFORMACIÓN DEL AUTOR}

Casas Patiño, Donovan

Correo: capo730211@yahoo.es 